\title{
HRTEMFringeAnalyzer a free python module for a automated analysis of fringe pattern in transmission electron micrographs
}

\author{
Ivo Alxneit*
}

February 18, 2020

\section{Introduction}

The human eye coupled with the human brain acts as an extremely powerful image analysis system capable of recognizing patterns or structures even in very noisy images. However, human based image analysis is also easily subjected to bias (i.e. we see what we want to see). Thus, it is often preferential to apply computer algorithms, not only because they allow for (fast) batch processing, but also because human bias is avoided. Even if these algorithms are not perfect, at least they introduce always the same bias.

Here, we report a Fourier transform based algorithm that allows to analyze and quantify the local crystallinity of samples imaged with a transmission electron microscope. By local crystallinity we denote the observation of extended regions with a more or less well defined fringe pattern. The local crystallinity is described by the lattice spacing $d$ of the structure and the direction $\phi$ of the corresponding lattice vector perpendicular to the fringes. The same definition of $d$ is used in the analysis of spot patterns. From the error $\sigma$ of the two quantities the coherence length of the structure (proportional to $\left.1 / \sigma_{d}\right)$ and the variability of the direction vector $\phi\left(\sigma_{\phi}\right)$ are determined. Maps of these indicators are obtained after processing the electron micrographs.

${ }^{*}$ Bioenergy and Catalysis Laboratory

Paul Scherrer Institute

CH-5232 Villigen PSI

Email: ivo.alxneit@psi.ch

phone: +41 (0)563104092 


\begin{abstract}
Standard Abstract: A python module (HRTEMFringeAnalyzer) is reported, to evaluate the local crystallinity of samples from high resolution TEM images in a mostly automated fashion. The user only selects the size of a square analyzer window and a step size which translates the window in the micrograph. Together they define the resolution of the results obtained. Regions where fringe patterns are visible are identified and their lattice spacing $d$ and direction $\phi$ as well as the corresponding mean errors $\sigma$ determined. $1 / \sigma_{d}$ is proportional to the coherence length of the structure while $\sigma_{\phi}$ is a measure of how well the direction of the fringes is defined. Maps of these four indicators are computed. The performance of the program is demonstrated on two very different samples: ill-crystalline carbon deposits on a coked $\mathrm{Ni} / \mathrm{LFNO}$ (reduced $\mathrm{LaFe}_{0.8} \mathrm{Ni}_{0.2} \mathrm{O}_{3 \pm \delta}$ ) catalyst and wellcrystallized nano particles of zinc doped ceria. In the latter case the automatic segmentation of large aggregates into individual crystalline domains is achieved by $\phi$ maps.

Lay Description: A python module (HRTEMFringeAnalyzer) is reported that evaluates the local crystallinity of samples from high resolution TEM images in a mostly automated fashion. Regions where fringe patterns are visible are identified and lattice spacing $d$ and direction $\phi$ are determined. The performance of the program is demonstrated on two very different samples: carbon deposits on a coked nickel catalyst and well crystallized nano particles of zinc doped ceria. The latter case demonstrates that an automatic identification of individual crystalline domains is achieved by $\phi$ maps.
\end{abstract}

Keywords: texture analysis, pyrocarbon, segmentation, nano particles, morphology 
After detailing the algorithm and its implementation, two application cases will be discussed: Visualizing the structure of carbon filaments observed in coked $\mathrm{Ni} / \mathrm{LFNO}$ (reduced $\mathrm{LaFe}_{0.8} \mathrm{Ni}_{0.2} \mathrm{O}_{3 \pm \delta}$ ) catalysts (Marcilly et al., 1970; Steiger et al., 2017) as well as using $\phi$ maps to segment zinc doped ceria nano particles (Lin et al., 2015a,b, 2016) into single crystalline domains.

Several methods to analyze local structures observed in transmission electron micrographs have been discussed in the literature. Seyring et al. (Seyring et al., 2009) describe a segmentation algorithm where HRTEM images are Fourier transformed to yield pattern similar to selected area diffraction patterns. A mask selects small regions along the diffraction rings observed, which are then back transformed and result in synthetic DF images. All synthetic DF images corresponding to a single diffraction ring, i.e. to the same fringe spacing, are superimposed and visualize the responsible nano particles from the HRTEM image. The resulting images can be segmented easily. Phase and amplitude information of Fourier transformed TEM micrographs were used to identify e.g. anti-phase boundaries (Hÿtch, 1997a,b). An overview of different algorithms to identify biological structures has recently been published (Püspöki et al., 2016). Algorithms discussed are based on the local gradient of the contrast, its Hessian, or based on wavelets. Notably, Fourier transform based methods are not mentioned. Fourier transform based band pass filtering has, however, been used as first step to extract the morphology of lattice fringes in pyrocarbon (Li et al., 2014). A different approach is based on convolution masks. Gradient and Vallyness operators (Pouliquen et al., 2005) can be implemented as convolution masks and were used to identify periodic structures. Another approach is based on FIR filters (Lyvers and Mitchell, 1988) but seems to be prone to noise. Most of these efforts seem to be dedicated to image enhancement or to specific questions and not to obtain a quantitative description of local structures. The results are generally images such as e.g. the ridge lines of complex fringe pattern observed in pyrocarbon (Li et al., 2014).

Several software approaches have been reported De Backer et al. (2016); Vasudevan et al. (2016); Nord et al. (2017) to quantitatively analyze the local structure of atomically resolved STEM data. These approaches can potentially give similar information as HRTEMFringeAnalyzer(Alxneit, 2018) for TEM data.

For the image processing suite Image J (Schindelin et al., 2012) two plugins, OrientationJ (Püspöki et al., 2016) and ImageJ-Directionality (Liu, 1991), are available to identify local periodic structures. Both seem to have been developed with biological samples in mind and did not yield satisfactory results on the micrographs discussed below. 


\section{Methodology}

\subsection{Algorithm}

Local information on crystallinity and the direction of the lattice vector can be obtained from the Fourier transform of a small region in a electron micrograph. Thus, the micrograph is probed by a window with dimensions $N \times N$ pixels to select a local region of interest (ROI) to be analyzed. The window should be larger than the period $d$ of the structure, i.e. it should be large enough to contain several fringes. At the same time it should be small enough to reveal local information such as the direction of the lattice vector, $\phi$, associated with the periodic structure. Choosing $N$ to be a power of 2 ensures an efficient calculation of the Fourier transform. The step size $s$ i.e. the number of pixels by which the window is shifted when the micrograph is analyzed, mostly defines the resolution of the result and can be much larger than a single pixel. Note, the the number of ROIs to be analyzed and therefore the computational cost is proportional to $1 / s^{2}$. To minimize computational cost while retaining a decent resolution a step size of the order of a single $d$-value is often appropriate. Each ROI analyzed provides a $d$-value, the direction $\phi$ of the lattice vector, and the corresponding errors, $\sigma_{d}$ and $\sigma_{\phi}$. Low resolution maps of these indicators are obtained and can be easily interpolated to the original size of the micrograph. The border region with a width of $N / 2$ pixels will be missing in the resulting maps as each ROI has to completely overlap with the micrograph. Five tuning parameters (marked in the following by tune) can be used to optimize the algorithm for the specific application.

The algorithm works as follows:

1. For each ROI selected, the image contrast is equalized by mapping it into the interval $[0,1]$. Then, the mean pixel count is subtracted to minimize the DC term in the Fourier transform.

2. The ROI is multiplied by a $2 \mathrm{D}$ Hanning window to prevent high frequency noise caused by the non-periodic cyclic boundaries. A Hanning window is used as it enforces zero pixel count at the boundaries while maintaining a high frequency resolution.

3. The $2 \mathrm{D}$ Fourier transform is calculated and quadrants in the $2 \mathrm{D}$ frequency matrix are flipped to shift the zero frequency to its center. Thus, the different frequencies are now located on circles with radius $f$ with the DC term as their center. For further processing the modulus squared, $I$, of the complex frequency components is used. 
4. Contributions by low frequencies (tune), by frequencies close to the Nyquist frequency (tune), and below a chosen noise level (tune) are removed.

5. (a) To determine the lattice spacing $d$ of a possible periodic structure a histogram $I(f)$ (azimuthal integration) is calculated.

(b) To obtain the direction $\phi$ of the lattice vector a histogram $I(\phi)$ (frequency integration) is calculated.

High frequency components lie on circles with larger geometrical radius, $r$, and are sampled more often than low frequency components. Thus, all data $I(f)=I(x, y)=I(r)$ are weighted by a factor of $1 / r^{2}$ with $r=\sqrt{x^{2}+y^{2}}$ and $x, y$ the indices of the quadrant-flipped FFT.

6. A Gaussian, $A e^{-4 \ln 2\left(\frac{x-x_{0}}{\sigma}\right)^{2}}$ plus an offset $y_{0}$ is then fitted to each of the histograms. The center value $x_{0}$ of the Gaussian is used as an estimate of $d$ and $\phi$, respectively. The width $\sigma_{\phi}$ is a measure how well the direction of the lattice vector is defined. On the other hand, $\sigma_{d}$ is a measure of how well the periodic structure is defined. It is inversely proportional to the coherence length. This interpretation follows the idea behind the Scherrer formula (Jenkins and Snyder, 1996) used in powder x-ray diffraction to obtain crystallite sizes from the peak width. $\sigma_{d}$ is influenced by $N$ because the size of the ROI typically is smaller than extent of the periodic structure. The scaling factor $4 \ln 2$ ensures that $\sigma$ corresponds to the FWHH (Full Width at Half Height) of the Gaussian. Only histograms where the maximum surpasses the mean bin count by a chosen threshold (tune) are considered to contain information and are fitted.

\subsection{Implementation}

The algorithm is implemented in Python3 because it provides a well developed ecosystem with many free libraries. HRTEMFringeAnalyzer(Alxneit, 2018) requires the quasi-standard libraries NumPy, (van der Walt et al., 2011) SciPy, (Jones et al., 2001-) and Matplotlib (Hunter, 2007). imageio is used to read the images. Additionally, using the Numba @ jit decorator for the evaluation of the Gaussian that is evaluated many times during the fit of each histogram results in a speed-up by a factor of about 4.5 of this function. Values such as $r^{2}=x^{2}+y^{2}, \phi=\arctan (y / x)$, or the 2D Hanning window are calculated once and passed on, as they depend only on the (constant) dimension of the ROI but not on its position within the micrograph. joblib 
is used to analyze individual rows of ROIs in parallel. This results in a decrease of the execution time by $48 \%, 71 \%, 76 \%$ on a 4 core ( 8 hyperthreads) Intel Xeon E3-1245 CPU when using 2, 4, 8 parallel jobs (see Fig. 1). As two threads (instead of one) are running per physical core, when eight parallel jobs are started, little additional benefit is realized compared to four parallel jobs.

\section{$2.3 \quad$ Limitations}

The key point of the algorithm is that a periodic structure inside a given ROI will result in a single peak in its Fourier transform. Thus, only the highest peak above the noise floor is analyzed. Whether a peak is identified depends on the contrast of structure and the $\mathrm{S} / \mathrm{N}$ ratio of the micrograph. The selection criterion could be optimized by the tuning parameters but is well behaved. Fitting a Gaussian to the data easily converges. Shape and definition of the peak depend on the bin width of the histograms that are rather large because only limited data is available. Thus the resolution of the fit is somewhat limited for $\phi$ and especially $\sigma_{\phi}\left(\approx 5^{\circ}\right)$. The analysis seems well behaved as reliable data or no data (no peak identified or insufficient quality of the fit) is returned. A very small value of $\sigma_{\phi}$ can erroneously be reported for structures with a very large period that conflicts with the size of the ROI (see Fig. S14).

If several periodic structures are present such as in e.g. overlapping crystals only the most intense peak in the Fourier transform is processed. The analysis might then alternate between the different peaks as the ROI is moved over the image which results in "fractured" regions in the maps.

\section{$3 \quad$ Examples}

Micrographs were collected with a JEOL JEM 2010 transmission electron microscope equipped with with a $\mathrm{LaB}_{6}$ cathode and operated at $200 \mathrm{keV}$. The samples were prepared on lacey carbon grids from sonicated dispersions in isopropanol. Images were recorded by a slow scan CCD camera $(4008 \times 2672$ pixels, Orius Gatan Inc.). The micrographs were preprocessed (X-ray signals removed) and then saved as 16-bit TIF files to maintain the full dynamic range provided by the CCD camera in Image J (Schindelin et al., 2012).

The Ni/LFNO sample was prepared by reduction $\left(600^{\circ} \mathrm{C}, 20 \mathrm{vol} \% \mathrm{H}_{2} / \mathrm{Ar}\right.$, $1 \mathrm{~h}$ ) of a $\mathrm{LaFe}_{0.8} \mathrm{Ni}_{0.2} \mathrm{O}_{3 \pm \delta}$ (LFNO) perovskite type metal oxide which was prepared using an amorphous citrate approach using metal nitrate precursors (Marcilly et al., 1970; Steiger et al., 2017). Subsequent coking was carried out 
in a plug flow reactor under $\mathrm{CO}_{2}$ methanation conditions $\left(430^{\circ} \mathrm{C}, 3000 \mathrm{ppm}\right.$ $\mathrm{C}_{2} \mathrm{H}_{4} / 32 \mathrm{vol} \% \mathrm{H}_{2} / 8 \mathrm{vol} \% \mathrm{CO}_{2} /$ rest $\mathrm{Ar}, 8 \mathrm{~h}$ ). TEM images of the coked $\mathrm{Ni} / \mathrm{LFNO}$ sample show Ni particles of a broad size range, most of them still attached to the support. The perovskite support crystallites form aggregates of various sizes. Only on some of these aggregates carbon structures are found. Most carbon structures do not exhibit a well defined morphology. Single, long, more or less straight filaments, wide bands, and cauliflower-like structures are observed. A typical example is reported in Fig. 2. Filaments still attached to a catalyst or support particle (top left) are observed. An unsupported $\mathrm{Ni}$ particle embedded in the carbon structures is visible near the center of the figure. Lattice fringes are observed in some part of the filaments but their location and orientation seem to be rather random.

In order to rationalize the observed carbon structures and to detect a possible hidden order of the crystalline regions HRTEMFringeAnalyzer was used to analyze the local crystallinity. The result of this analysis for the TEM image of Fig. 2 using a probing ROI of $128 \times 128$ pixels and a step size of 8 pixels is reported in Fig. 3. These maps semi-transparently superimposed on the original TEM image are reported in the Supplementary Material (Figs. S1-S4).

A clear ordering is observed in the direction $(\phi)$ map of Fig. 3. Where present, the direction of the lattice fringes is close to parallel to the long axis of the filaments, i.e. the lattice vector $d$ is only slightly inclined relative to their surface. In the spinal region of the filaments fringes are less prominent but seem to run perpendicular the the axis. Thus the structure looks similar to stacked cups. The same structural features are observed on the cauliflower-like structure at the center top of Fig. 2. Directions are well defined with $\sigma_{\phi}$ values generally around $10^{\circ}$. Regions where the lattice spacing $d$ could be determined are rather sparse and generally small. Also the corresponding coherence lengths are very small compared to the ones observed for the well crystalline material analyzed in Fig. 5 which is to be expected when comparing the TEM micrographs presented in Figs. 2 and 4 . Where the lattice spacing $d$ could be determined in Fig. 2, values in the range of 17 pixels $(0.3 \mathrm{~nm})$ were observed. These are in good agreement with fringe spacings $d_{002}$ observed in various forms of graphitic carbon (Li et al., 2014).

The ceria samples studied (see Fig. 4) consist of well crystallized nano particles as apparent from the well developed lattice fringes. Rather than individual single crystalline particles, relatively large agglomerates are observed. The extraction of a particle size distribution from a series of such images would pose rather tedious manual work. Regions covered by lattice fringes of a common lattice vector identify individual single crystalline nano particles. These have to be individually marked and then analyzed. 
An automatic segmentation algorithm acting on the original micrograph seems difficult. A directionality-map (see Fig. 5, bottom left) calculated by HRTEMFringeAnalyzer (probing ROI $128 \times 128$ pixels, step size 8 pixels) achieves this segmentation. Individual nano particles can be clearly identified based on their color (direction $\phi$ of the lattice fringes) and segmentation is thus achieved. A few dilation - erosion passes would clean up the image by removing small holes and islands. $\sigma_{\phi}$ (see Fig. 5, bottom right) is small, below $10^{\circ}$, in regions where the fit converged. The numerical values of $\sigma_{\phi}$ should be considered only approximate as the bin width of the $I(\phi)$ histogram is $5^{\circ}$ and only coarsely describes the peak shape.

Regions with a lattice spacing in the interval $[18,22]$ (see blue regions in Fig. 5 top, left) overlap with regions with a high coherence and a well defined direction of the lattice vector, i.e. the large, well crystalline regions. Considering only this subset an average $d$-value of 20.308 pixels or $0.315 \mathrm{~nm}$ is found close the values of $d_{111}=0.294 \mathrm{~nm}$ and $d_{111}=0.298 \mathrm{~nm}$ determined elsewhere from HRTEM micrographs (Lin et al., 2015a). Overlapping particles are difficult to resolve: The Fourier transform of the ROI exhibits two peaks in this case, one for each particle. The algorithm, at present, does only processes the most intense peak which might randomly alternate between the two fringe patterns as the ROI is shifted. Moiré patterns, however, usually do not represent a problem as their lattice spacings are much larger than the spacing of lattice planes. The user supplied size of the analysis window is generally too small to recognize them. The moiré pattern visible in the topleft of Fig. 4 with direction vector pointing towards right exemplifies this. Again, the maps semi-transparently superimposed on the TEM image are reported in the Supplementary Material Figs. S5-S8.

The analysis presented can easily be extended to mixtures where individual phases can be selected based on their lattice spacings. Similarly, approximate grain geometries could be deduced from a statistical analysis of materials that exhibit lattice fringes with different spacings.

\section{Conclusions}

A simple, memory efficient, and reasonably fast algorithm is presented that analyzes the local crystallinity (lattice spacing and direction with corresponding error values) from TEM micrographs in a mostly automated way. The user only selects the size of a square window used as a probe in the analysis and a step size by which the probe scans the image. A few internal "tuning knobs" are all connected to the statistical indicators used to select the peaks in the power spectrum used in the analysis. They can be optimized to specific 
problems, but the values used in the implementation HRTEMFringeAnalyzer work generally well and have been used throughout this report.

The algorithm is demonstrated with two very different application cases: carbon structures observed on coked Ni/LFNO catalysts and Zn doped ceria. These examples cover both, ill and well crystallized materials and demonstrate that the algorithm can be applied to very different samples. It works well independent of magnification as long as the dimension of the probe window is selected to cover at least around five periods of the fringe pattern. Additional applications, such as the analysis of complex mixtures or the determination of the external geometry of crystallites are also mentioned.

\section{Supplementary Material}

Supplementary Material containing maps overlayed on micrographs (Figs. S1S8), intermediary results of several ROI's processed by HRTEMFringeAnalyzer, and the original 16bit TIF files of Figs. 2 and 4 is available from the journal's home page. https://github.com/ialxn/HRTEMFringeAnalyzer provides free access to the software discussed.

\section{Acknowledgements}

The author gratefully acknowledges Patrick Steiger providing coked Ni/LFNO samples, Dr. Elisabeth Müller for her continuous support and time for discussions, and one of the reviewers for the image of "stacked cups".

\section{References}

I. Alxneit. HRTEMFringeAnalyzer - a python module to evaluate the local crystallinity of samples from high resolution TEM images in a mostly automated fashion. Available at https://github.com/ialxn/HRTEMFringeAnalyzer, February 2018.

A. De Backer, K. van den Bos, W. Van den Broek, J. Sijbers, and S. Van Aert. Statstem: An efficient approach for accurate and precise model-based quantification of atomic resolution electron microscopy images. Ultramicroscopy, 171:104 - 116, 2016. doi: 10.1016/j.ultramic.2016.08.018.

J. D. Hunter. Matplotlib: A 2d graphics environment. Comput. Sci. Eng., 9 (3):90-95, 2007. 
M. J. Hÿtch. Geometric phase analysis of high resolution electron microscope images. Scanning Microsc., 11:53-66, 1997a.

M. J. Hÿtch. Analysis of variations in structure from high resolution electron microscope images by combining real space and fourier space information. Microscopy Microanalysis Microstructures, 8(1):41-57, $1997 \mathrm{~b}$.

R. Jenkins and R. L. Snyder. Introduction to X-ray powder diffractometry. Chemical analysis: v. 138. New York : Wiley, 1996. doi: $10.1002 / 9781118520994$.

E. Jones, T. Oliphant, P. Peterson, et al. SciPy: Open source scientific tools for Python, 2001-. URL http://www.scipy.org/.

M. Li, H. Zhao, L. Qi, and H. Li. Analysis techniques of lattice fringe images for quantified evaluation of pyrocarbon by chemical vapor infiltration. Microsc. Microanal., 20(5):1591-1600, 2014. doi: 10.1017/S143192761400169X.

F. Lin, I. Alxneit, and A. Wokaun. Structural and chemical changes of Zn-doped $\mathrm{CeO}_{2}$ nanocrystals upon annealing at ultra-high temperatures. CrystEngComm, 17(7):1646-1653, 2015a. doi: 10.1039/c4ce02202e.

F. Lin, R. Delmelle, T. Vinodkumar, B. M. Reddy, A. Wokaun, and I. Alxneit. Correlation between the structural characteristics, oxygen storage capacities and catalytic activities of dual-phase Zn-modified ceria nanocrystals. Catal. Sci. Technol., 5(7):3556-3567, 2015b. doi: 10.1039/c5cy00351b.

F. Lin, V. A. Samson, A. O. Wismer, D. Grolimund, I. Alxneit, and A. Wokaun. Zn-modified ceria as a redox material for thermochemical $\mathrm{H}_{2} \mathrm{O}$ and $\mathrm{CO}_{2}$ splitting: effect of a secondary $\mathrm{ZnO}$ phase on its thermochemical activity. CrystEngComm, 18(14):2559-2569, 2016. doi: 10.1039/C6CE00430J.

Z.-Q. Liu. Scale space approach to directional analysis of images. Appl. Optics, 30(11):1369-1373, 1991. doi: 10.1364/AO.30.001369.

E. P. Lyvers and O. R. Mitchell. Precision edge contrast and orientation estimation. IEEE Transactions on Pattern Analysis and Machine Intelligence, 10(6):927-937, Nov 1988. doi: 10.1109/34.9114.

C. Marcilly, P. Courty, and B. Delmon. Preparation of highly dispersed mixed oxides and oxide solid solutions by pyrolysis of amorphous organic precursors. J. Am. Ceram. Soc., 53(1):56-57, 1970. 
M. Nord, P. E. Vullum, I. MacLaren, T. Tybell, and R. Holmestad. Atomap: a new software tool for the automated analysis of atomic resolution images using two-dimensional gaussian fitting. Adv. Struct. Chem. Imag., 3(1):9, 2017. doi: 10.1186/s40679-017-0042-5.

F. L. Pouliquen, J.-P. D. Costa, C. Germain, and P. Baylou. A new adaptive framework for unbiased orientation estimation in textured images. Pattern Recognition, 38(11):2032 - 2046, 2005. doi: 10.1016/j.patcog.2005.05.004.

Z. Püspöki, M. Storath, D. Sage, and M. Unser. Transforms and operators for directional bioimage analysis: A survey. Advances in Anatomy Embryology and Cell Biology, 219:69-93, 2016. doi: 10.1007/978-3-319-28549-8_3.

J. Schindelin, I. Arganda-Carreras, E. Frise, V. Kaynig, M. Longair, T. Pietzsch, S. Preibisch, C. Rueden, S. Saalfeld, B. Schmid, J.-Y. Tinevez, D. J. White, V. Hartenstein, K. Eliceiri, P. Tomancak, and A. Cardona. Fiji: an open-source platform for biological-image analysis. Nat Meth, 9(7): 676-682, July 2012. doi: 10.1038/nmeth.2019.

M. Seyring, X. Song, A. Chuvilin, U. Kaiser, and M. Rettenmayr. Characterization of grain structure in nanocrystalline gadolinium by high-resolution transmission electron microscopy. J. Mater. Res., 24(2):342-346, 2009. doi: $10.1557 /$ jmr.2009.0071.

P. Steiger, R. Delmelle, D. Foppiano, L. Holzer, A. Heel, M. Nachtegaal, O. Kröcher, and D. Ferri. Structural reversibility and nickel particle stability in lanthanum iron nickel perovskite-type catalysts. ChemSusChem, 10(11):2505-2517, 2017.

S. van der Walt, S. C. Colbert, and G. Varoquaux. The numpy array: A structure for efficient numerical computation. Computing in Science Engineering, 13(2):22-30, March 2011. doi: 10.1109/MCSE.2011.37.

R. K. Vasudevan, M. Ziatdinov, S. Jesse, and S. V. Kalinin. Phases and interfaces from real space atomically resolved data: Physics-based deep data image analysis. Nano Letters, 16(9):5574-5581, 2016. doi: 10.1021/acs.nanolett.6b02130. 


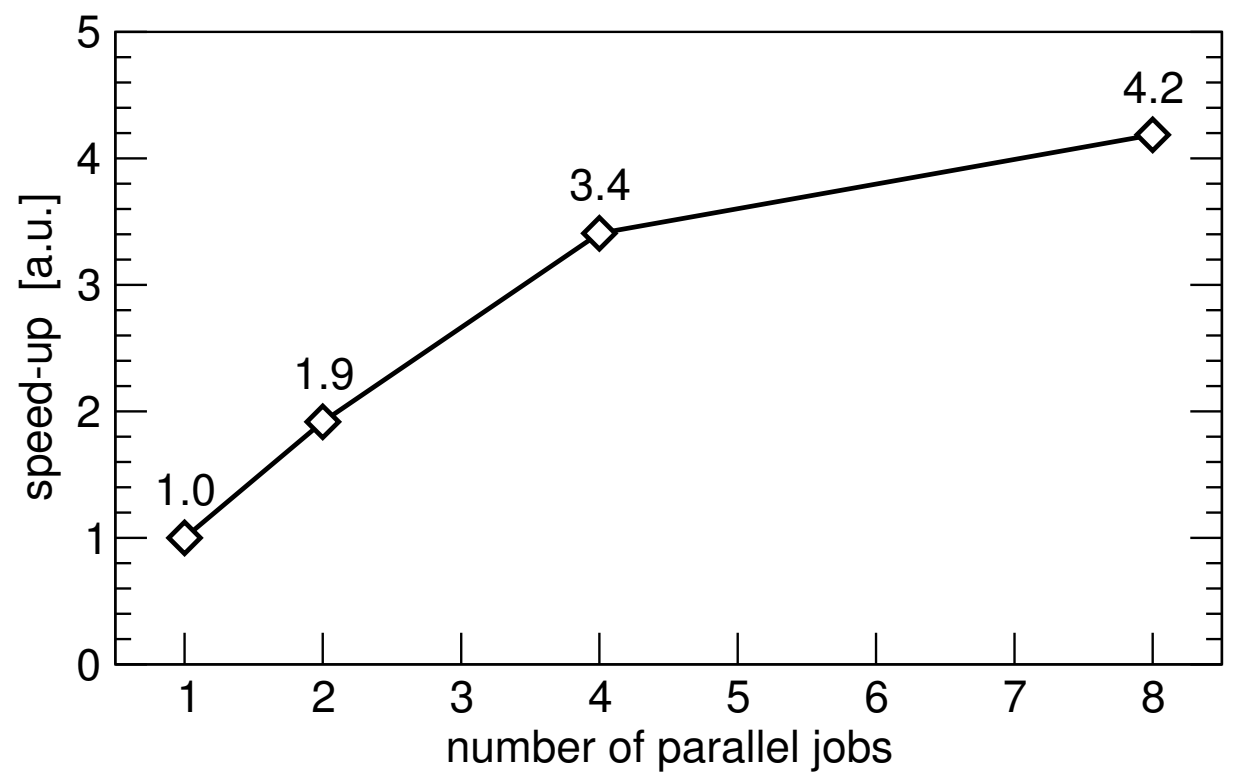

Figure 1: Scaling of execution time of HRTEMFringeAnalyzer with number of parallel jobs obtained on a 4 core (8 hyperthreads) Intel Xeon E3-1245 CPU. 
carbon.pdf

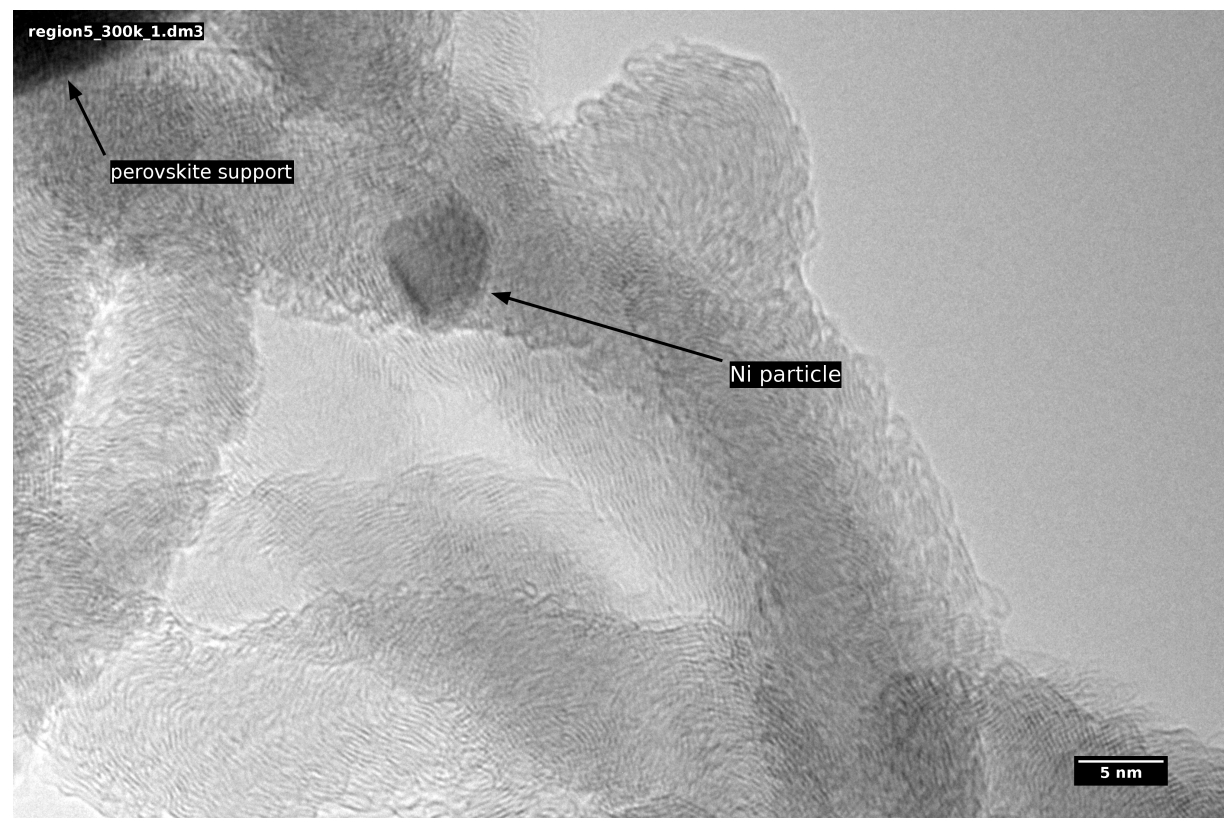

Figure 2: TEM image $(\mathrm{Mag}=300 \mathrm{k})$ of carbon filaments observed on coked $\mathrm{Ni} /$ LFNO. The original, full resolution tif-image is provided as Supplementary Material. 

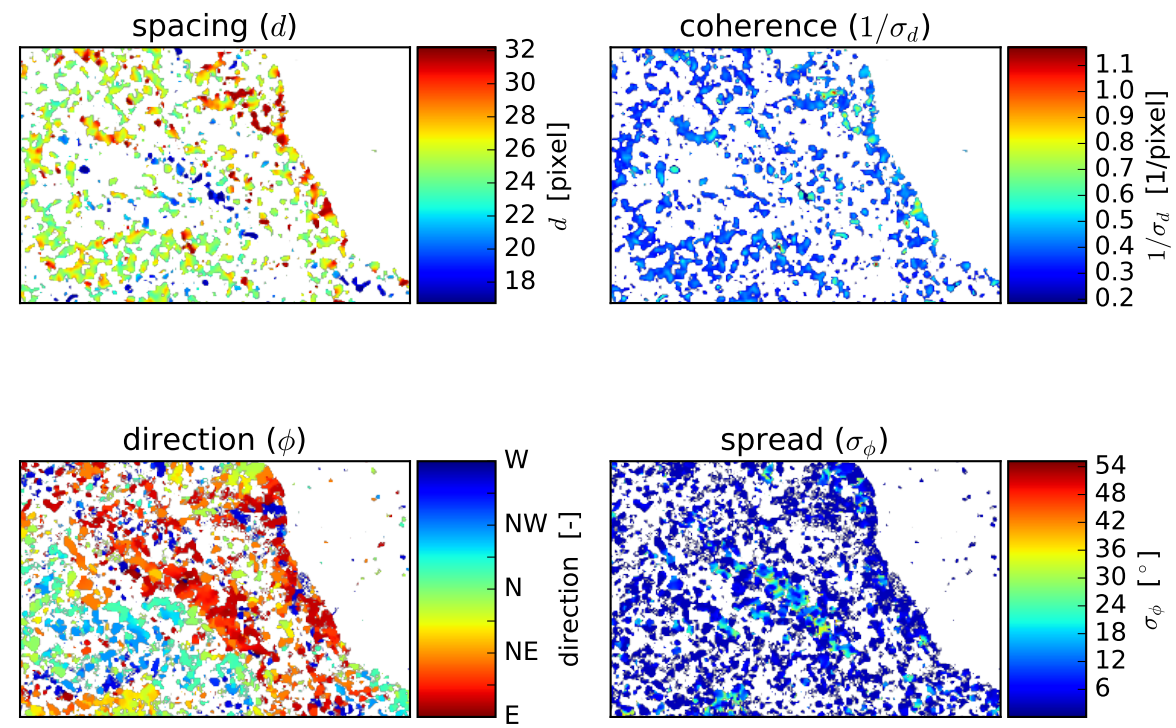

Figure 3: Local crystallinity analysis $(128 \times 128$ ROI, 8 pixel step size $)$ of the micrograph of Fig. 2. Top: lattice spacing $d$ (left) and coherence length $1 / \sigma_{d}$ (right). Bottom: direction $\phi$ of lattice vector (left) and $\sigma_{\phi}$ (right). 


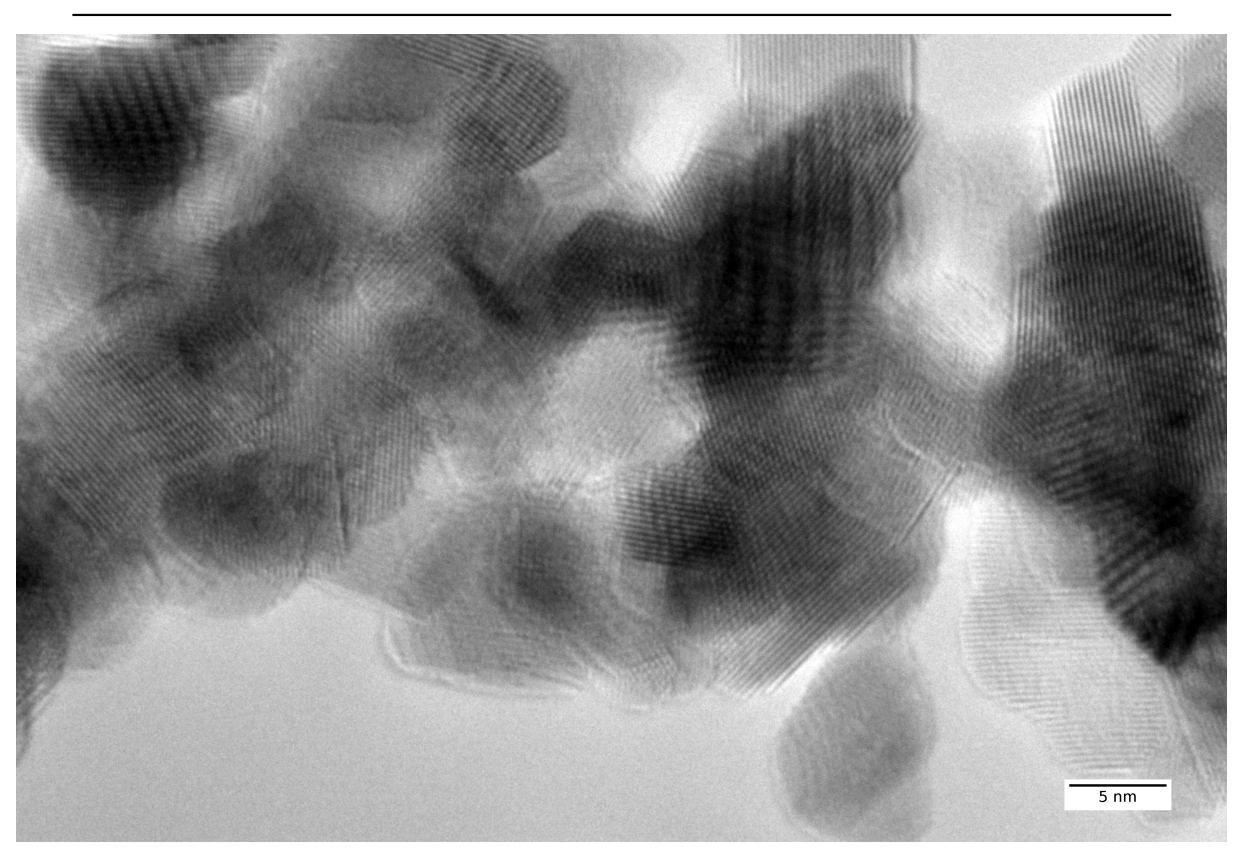

Figure 4: TEM image $(\mathrm{Mag}=400 \mathrm{k})$ of well crystalline ceria nano particles. The original, full resolution tif-image is provided as Supplementary Material. 

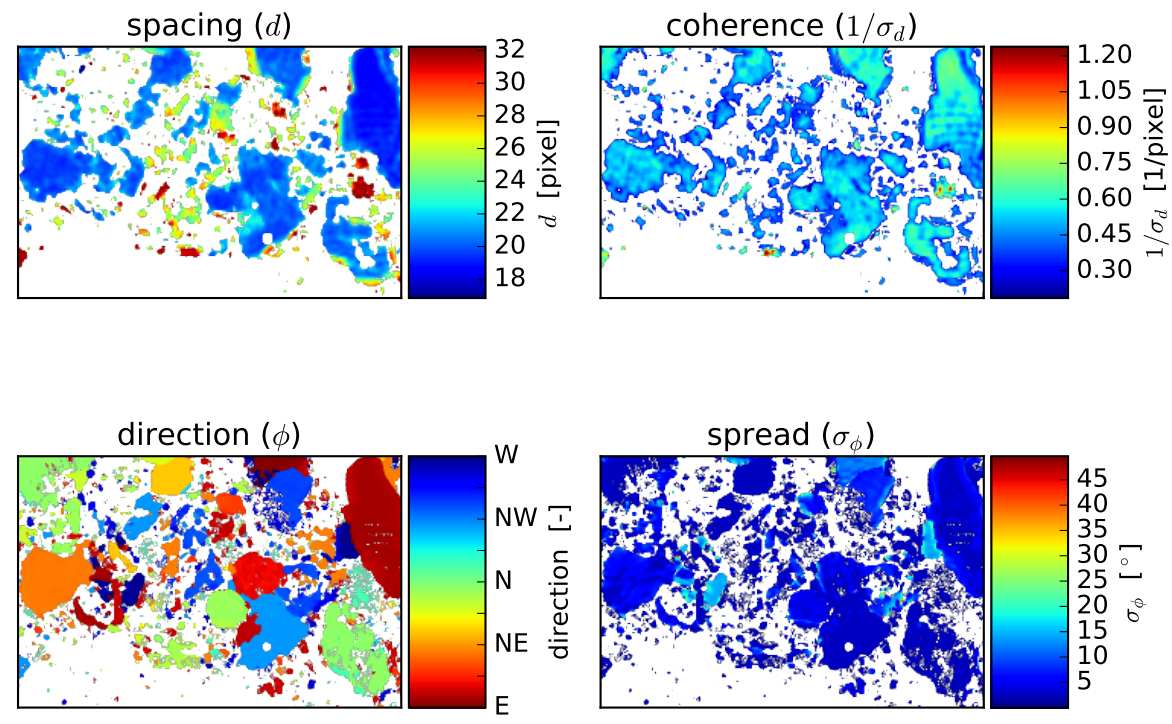

Figure 5: Local crystallinity analysis $(128 \times 128 \mathrm{ROI}, 8$ pixel step size $)$ of the micrograph of Fig. 4. Top: lattice spacing $d$ (left) and coherence length $1 / \sigma_{d}$ (right). Bottom: direction $\phi$ of lattice vector (left) and $\sigma_{\phi}$ (right). 